\title{
Double Circle
}

National Cancer Institute

\section{Source}

National Cancer Institute. Double Circle. NCI Thesaurus. Code C48339.

The shape of two circles joined together, and usually overlapping. 